Обоснование. Гиперпролактинемия является одним из распространенных гипоталамо-гипофизарно-эндокринных нарушений у женщин репродуктивного возраста, с наибольшей частотой в возрасте 25-44 лет. Помимо влияния на репродуктивную систему, важно изучение эффектов пролактина на различные звенья метаболизма. Имеющиеся данные указывают на то, что результат воздействия пролактина на метаболизм зависит от его уровня. В связи с этим актуально изучение связи разных уровней пролактина с антропометрическими параметрами, показателями липидного и углеводного обменов у женщин молодого возраста.

Цель. Изучить частоту метаболического синдрома (MC) и его отдельных компонентов у женщин 25-45 лет с различными уровнями пролактина.

Meтоды. Дизайн работы - поперечное исследование. Обследована случайная популяционная подвыборка женщин 25-45 лет. Исключались беременные и осуществляющие грудное вскармливание, женщины с макропролактиномой, принимающие нейролептики. Проведен сбор информации с помощью структурированного вопросника, включающего, в том числе, вопросы по наличию беременностей, родов, нарушений менструального цикла, выполнены клинический осмотр, антропометрические измерения, биохимические и гормональные анализы крови. Проведена статистическая обработка данных.

Результаты. С учетом критериев включения и исключения в настоящем анализе представлены данные 401 женщи-

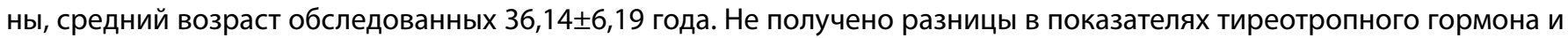
пролактина (ПРЛ) в возрастных группах 25-34 и 35-45 лет. По данным опроса, сопоставима частота заболеваний щитовидной железы в изучаемых группах. Каждая пятая женщина указала на нарушения менструального цикла. Среди женщин 25-45 лет менее благоприятные метаболические показатели имеют женщины с низко-нормальными значениями ПРЛ (медиана (Ме)=4,49 [3,52; 5,41] нг/мл). МС выявлен у 28\%, с преимущественным увеличением частоты абдоминального ожирения - 55\%, повышения уровня холестерина липопротеидов низкой плотности - 63\%. Женщины, имеющие высокие значения ПРЛ (Ме=41,35 [34,78; 45,88] нг/мл), также имеют неблагоприятный метаболический профиль: МС выявлен у 47\%, абдоминальное ожирение - 56\%, артериальная гипертензия - 39\%.

Заключение. У женщин 25-45 лет низкие и высокие значения ПРЛ чаще связаны с метаболическим нездоровьем. Значения ПРЛ от 7,8 до 28 нг/мл, т.е. условно определяемые как нормальные, высоконормальные и на уровне умеренной гиперпролактинемии, способствуют поддержанию благоприятного метаболического профиля. При решении вопроса о лечении женщин с гиперпролактинемией неопухолевой этиологии важно оценивать метаболический статус, расширяя свои представления о ПРЛ как о гормоне, связанном только с лактацией и с гипофизарно-гонадной осью.

КЛЮЧЕВЫЕ СЛОВА: пролактин; гиперпролактинемия; метаболический синдром; ожирение; липиды.

\title{
THE FREQUENCY OF METABOLIC SYNDROME AND ITS INDIVIDUAL COMPONENTS IN WOMEN AGED 25-45 YEARS, DEPENDING ON THE LEVEL OF PROLACTIN
}

(c) Oksana D. Rymar*, Svetlana M. Voevoda, Elena V. Shachtshneider, Ekaterina M. Stakhneva, Svetlana V. Mustafina, Lilia V. Shcherbakova

Institute of Cytology and Genetics, Siberian Branch of the Russian Academy of Sciences, Novosibirsk, Russia

BACKGROUND: Hyperprolactinemia is one of the most common hypothalamic-pituitary-endocrine disorders in women of reproductive age, with the highest frequency at the age of 25-44 years. In addition to influencing the reproductive system, it is important to study the effects of prolactin (PRL) on various metabolic links. Available data indicate that the effect of PRL on metabolism depends on its level. In this regard, the study of the relationship of different levels of PRL with anthropometric parameters, indicators of lipid and carbohydrate metabolism in young women is relevant.

AIM: To study the frequency of metabolic syndrome (MS) and its individual components in women aged 25-45 years with different levels of prolactin.

MATERIALS AND METHODS: Work design - cross-sectional research. A randompopulationsample of women 25-45 agedwas examined. Pregnant and breastfeeding women with macroprolactinoma, and taking antipsychotics were excluded. Information was collected using a structured questionnaire, including, but not limited to, the presence of pregnancies, childbirth, menstrual irregularities, and a clinical examination, anthropometric measurements, biochemical and hormonal blood analyzes were performed. Statistical data processing was carried out. 
RESULTS: According to the inclusion and exclusion criteria, this analysis presents data from 401 women, the average age of the examibed was $36.14 \pm 6.19$ years. There was no difference in the levels of thyroid-stimulating hormone and prolactin (PRL) in the age groups of 25-34 and 35-45 years. According to the survey, the incidence of thyroid diseases in the studied groups is comparable. Every fifth woman indicated menstrual irregularities. Among women $25-45$ years old, women with low-normal PRL values ( $\mathrm{Me}=4.49[3.52 ; 5.41] \mathrm{ng} / \mathrm{ml}$ ) have more unfavorable metabolic indicators. Metabolic syndrome (MS) was detected in $28 \%$, with a predominant increase in the frequency of abdominal obesity - 55\%, hypercholesterolemic $\mathrm{LDL}-63 \%$. Women with high PRL (Me $=41.35[34.78 ; 45.88] \mathrm{ng} / \mathrm{ml}$ ) also have an unfavorable metabolic profile: MS was detected in $47 \%$, abdominal obesity - $56 \%$, hypertension - $39 \%$.

CONCLUSIONS: In women 25-45 years old, low and high PRL values are more often associated with metabolic ill health. PRL values are from 7.8 to $28 \mathrm{ng} / \mathrm{ml}$, i.e. conditionally defined as normal, highly normal and at the level of moderate hyperprolactinemia contribute to the maintenance of a favorable metabolic profile. When deciding on the treatment of women with non-tumor etiology hyperprolactinemia, it is important to assess the metabolic status, expanding their understanding of PRL as a hormone associated only with lactation and with the pituitary-gonad axis.

KEYWORDS: prolactin; hyperprolactinemia; metabolic syndrome; obesity; lipids.

\section{ОБОСНОВАНИЕ}

Гиперпролактинемия является одним из наиболее распространенных гипоталамо-гипофизарно-эндокринных нарушений среди женщин репродуктивного возраста. В исследовании PROLEARS показано, что самая высокая частота гиперпролактинемии была обнаружена у женщин в возрасте 25-44 лет [1]. Помимо влияния на репродуктивную систему, важно изучение эффектов пролактина (ПРЛ) на различные звенья метаболизма [2]. Обширные клинические и экспериментальные данные указывают на то, что результат воздействия ПРЛ на метаболизм зависит от уровня циркулирующего гормона. По мнению большинства исследователей, патологическая гиперпролактинемия с чрезвычайно высокими значениями, обусловленная пролактиномой, связана с ожирением, нарушением толерантности к глюкозе и инсулинорезистентностью как у мужчин, так и у женщин [36]. Не так много исследований, изучающих влияние ПРЛ, находящегося в пределах и выше физиологического порога, на метаболический гомеостаз. В немногочисленных работах показано, что «более высокие» (в пределах и выше физиологического диапазона 25 мкг/л, или 525 мЕД/л) уровни ПРЛ способствуют благоприятному метаболическому гомеостазу при диабете и метаболических изменениях, вызванных ожирением [7]. В разные годы в ряде клинических и экспериментальных работ было показано, что низкие уровни ПРЛ связаны с неблагоприятным метаболическим профилем [8-10]. Нужно отметить, что метаболические последствия низконормальных значений и гиперпролактинемии у женщин молодого возраста в большей степени мало изучены. Отсутствуют исследования распространенности метаболического синдрома (МС) и его компонентов у молодых женщин с разным уровнем ПРЛ неопухолевой природы. В связи с этим актуально изучение связи разных уровней ПРЛ с антропометрическими параметрами, показателями липидного и углеводного обменов у женщин молодого возраста.

\section{ЦЕЛЬ}

Изучить частоту MC и его отдельных компонентов у женщин 25-45 лет с различными уровнями пролактина.

\section{МАТЕРИАЛЫ И МЕТОДЫ}

Место и время проведения исследования

Место проведения. Исследование проводилось в скрининг-центре Научно-исследовательского института терапии и профилактической медицины филиала Федерального исследовательского центра Института цитологии и генетики СО РАН. Гормональное и биохимическое исследование крови выполнено в лаборатории клинических биохимических и гормональных исследований терапевтических заболеваний НИИТПМ - филиала ИЦиГ СО РАН, имеющей стандартизацию по внутреннему и внешнему федеральному контролю качества.

Продолжительность исследования. Набор материала продолжался в период с 2013 по 2017 гг.

Изучаемые популяции (одна или несколько)

Обследована одна популяция.

Критерии включения: в исследование были включены женщины 25-45 лет.

Критерии исключения: беременные и осуществляющие грудное вскармливание, наличие макропролактиномы или прием нейролептиков.

Способ формирования выборки из изучаемой популяции (или нескольких выборок из нескольких изучаемых популяций)

Данное исследование выполнено на материале репрезентативной выборки из неорганизованной популяции женщин 25-45 лет, обследованных в рамках бюджетной темы «Эпидемиологический мониторинг состояния здоровья населения и изучение молекулярно-генетических и молекулярно-биологических механизмов развития распространенных терапевтических заболеваний в Сибири для совершенствования подходов к их диагностике, профилактике и лечению». Описание формирования выборки представлено ранее [11]. Расчет необходимого объема подвыборки осуществляли с учетом литературных данных о распространенности гиперпролактинемии у женщин молодого возраста [1]. Объем подвыборки рассчитан по формуле [12]:

$$
N=15,4 \times(p \times(1-p)) / W^{2},
$$


где: $\mathrm{p}$ - ожидаемое значение вероятности случайного события; W - ширина доверительного интервала для значения вероятности. По этой формуле минимальный объем изучаемой подвыборки молодых женщин составил 246 человек. Проведена процедура формирования простой случайной подвыборки с помощью генератора случайных чисел.

\section{Дизайн исследования}

Проведено одномоментное наблюдательное одноцентровое поперечное исследование.

\section{Описание медицинского вмешательства}

Проведен сбор информации с помощью структурированного опросника, включающего, в том числе, вопросы по наличию беременностей, родов, нарушений менструального цикла, привычке курения. Всем женщинам проведены антропометрические измерения (рост, вес с расчетом индекса массы тела (ИМТ), окружность талии (ОТ)). Измерение роста проводили стоя, без верхней одежды и обуви, на стандартном ростомере с точностью 0,5 см. Массу тела определяли без верхней одежды и обуви, на стандартных рычажных весах, прошедших метрологический контроль (точность измерения составляла 0,1кг). Измерение АД проводилось трехкратно. Проводился забор крови из локтевой вены утром натощак через 12 ч после приема пищи. После центрифугирования сыворотку хранили в низкотемпературной камере $\left(-70^{\circ} \mathrm{C}\right)$. Были определены показатели глюкозы, общего холестерина (ОХС), триглицеридов (ТГ), ХС липопротеинов высокой плотности (ХС-ЛПВП), ПРЛ и тиреотропного гормона (ТТГ) в сыворотке крови.

\section{Основной исход исследования}

В репрезентативной выборке женщин 25-45 лет с разным уровнем пролактина неопухолевого генеза изучены частота МС и его отдельных компонентов.

\section{Дополнительные исходы исследования}

Проанализированы данные о нарушениях менструального цикла, наличии заболеваний щитовидной железы, табакокурении; их ассоциации с ПРЛ среди обследованных женщин. Изучены показатели ПРЛ при разных значениях компонентов МС и наличии МС у женщин 25-45 лет.

\section{Анализ в подгруппах}

Участницы исследования были разделены на возрастные подгруппы 25-34 и 35-45 лет, которым даны клинико-лабораторные характеристики. Дополнительно для изучения метаболических показателей все женщины были разделены на 4 подгруппы с разным уровнем ПРЛ (по 25\% в каждой).

\section{Методы регистрации исходов}

Наличие нарушений менструального цикла, заболеваний щитовидной железы в анамнезе, привычки курения оценивали с помощью анализа данных структурированного вопросника. Наличие МС устанавливалось на основании критериев американского руководства NCEP ATP III (National Cholesterol Education Program Adult Treatment Panel III, 2001) [13], Всероссийского научного общества кардиологов (ВНОК, 2009, второй пересмотр) [14]. NCEP ATP III (2001) - три и более из нижеперечисленных компонентов: ОТ больше 88 см у женщин, ТГ $\geq 1,7$ ммоль/л, ХС-ЛВП <1,3 ммоль/л у женщин, АД $\geq 130 / 85$ мм рт. ст., содержание глюкозы в крови $\geq 6,1$ ммоль/л. BНОК (2009) ОТ больше 80 см у женщин плюс наличие двух из следующих критериев: АД $\geq 130 / 85$ мм рт. ст., ТГ $\geq 1,7$ ммоль/л, ХС-лПВП <1,2 ммоль/л у женщин, ХС-лПНП > 3,0 ммоль/л, глюкоза плазмы крови $\geq 6,1$ ммоль/л.

\section{Статистический анализ}

Методы статистического анализа данных. Статистическую обработку результатов проводили в программе SPSS forWindows (v.13), проведены автоматизированная проверка базы данных и статистический анализ. Характер распределения непрерывных показателей оценивался по тесту Колмогорова-Смирнова. Проведенная оценка указывает на наличие у всех непрерывных показателей ненормального распределения ( $p>0,05)$. Данные представлены как абсолютные (n) и относительные величины (\%), а также как $\mathrm{M} \pm \mathrm{SD}$, где $\mathrm{M}$ - среднее арифметическое значение, SD - стандартное отклонение, Me (25; 75), где Ме - медиана, 25-й и 75-й процентили. Изучаемые показатели представлены в квартилях ПРЛ. Сравнение двух независимых групп по количественным признакам с ненормальным распределением произведено с помощью непараметрического критерия МаннаУитни, внутригрупповые корреляции признаков оценивались при расчете коэффициента ранговой корреляции Спирмена. Оценка ассоциаций признаков проводилась с помощью однофакторного анализа с использованием таблиц сопряженности, также оценивалось отношение шансов (ОШ) с доверительным интервалом (ДИ). Долевое различие признаков вычислялось с помощью критерия $X^{2}$ Пирсона. Различия считались статистически значимыми при $\mathrm{p}<0,05$.

\section{Этическая экспертиза}

Исследование одобрено локальным этическим комитетом НИИТПМ - филиал ИЦиГ СО РАН протокол № 10 от 21.01.2014 г. Перед началом исследования все участники подписали информированное согласие.

\section{РЕЗУЛЬТАТЫ}

Объекты (участники) исследования

С учетом критериев включения и исключения в настоящем анализе представлены данные 401 женщины,

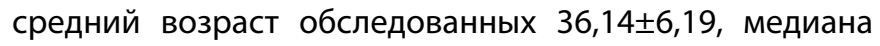
$36,25[30,88 ; 41,54]$ года.

\section{Основные результаты исследования}

Основные характеристики обследованных женщин представлены в таблицах 1, 2. Среди женщин с возрастом (во 2-й возрастной группе по отношению к 1-й) наблюдается статистически значимое увеличение веса, ИМТ, ОТ, ОХС, ХС-ЛПНП, ТГ, глюкозы плазмы натощак, систолического и диастолического артериального давления. Во 2-й возрастной группе выше частота избыточной массы тела и ожирения, абдоминального ожирения (АО), артериальной гипертонии (АГ), гиперхолестеринемии и повышенного уровня ХС-ЛПНП (гиперХС-ЛПНП), 


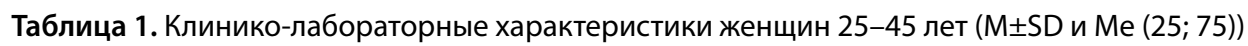

\begin{tabular}{|c|c|c|c|c|c|}
\hline & \multirow{2}{*}{ Показатели } & \multirow{2}{*}{ Вся выборка (n=401) } & \multicolumn{2}{|c|}{ Возрастная группа, лет } & \multirow{2}{*}{$\mathbf{p}$} \\
\hline & & & $25-34(n=177)$ & $35-44(n=224)$ & \\
\hline \multicolumn{2}{|c|}{ Возраст, лет } & $\begin{array}{c}36,14 \pm 6,19 \\
36,25(30,88 ; 41,54)\end{array}$ & $\begin{array}{c}30,15 \pm 2,96 \\
30,25(28 ; 32,67)\end{array}$ & $\begin{array}{c}40,88 \pm 3,28 \\
41,17(38,02 ; 43,58)\end{array}$ & $<0,001$ \\
\hline \multicolumn{2}{|c|}{ Рост, CM } & $\begin{array}{c}164,66 \pm 6,20 \\
164,51(160,0 ; 168,55)\end{array}$ & $\begin{array}{c}165,21 \pm 5,95 \\
165,52(161,25 ; 170,01)\end{array}$ & $\begin{array}{c}164,23 \pm 6,38 \\
164,01(160,02 ; 168,03)\end{array}$ & $\begin{array}{l}0,116 \\
0,073\end{array}$ \\
\hline \multicolumn{2}{|c|}{ Вес, кг } & $\begin{array}{c}69,65 \pm 16,81 \\
65,95(57,55 ; 76,95)\end{array}$ & $\begin{array}{c}67,51 \pm 17,46 \\
64,20(55,20 ; 72,75)\end{array}$ & $\begin{array}{c}71,35 \pm 16,11 \\
68,20(59,50 ; 79,0)\end{array}$ & $\begin{array}{l}0,023 \\
0,002\end{array}$ \\
\hline \multicolumn{2}{|c|}{ ИМТ, кг/м² } & $\begin{array}{c}25,67 \pm 5,98 \\
24,29(21,63 ; 28,34)\end{array}$ & $\begin{array}{c}24,69 \pm 6,04 \\
23,43(20,45 ; 27,0)\end{array}$ & $\begin{array}{c}26,45 \pm 5,82 \\
25,33(22,21 ; 29,27)\end{array}$ & $<0,001$ \\
\hline \multirow{4}{*}{$\begin{array}{l}\stackrel{\rho}{\Sigma} \\
\stackrel{E}{E}\end{array}$} & $<18,5 \mathrm{Kг} / \mathrm{M}^{2}$ & $17 / 4,3$ & $16 / 9,0$ & $1 / 0,4$ & $<0,001$ \\
\hline & $18,5-24,9 \mathrm{kг} / \mathrm{M}^{2}$ & $202 / 50,5$ & $96 / 54,2$ & $106 / 47,5$ & 0,170 \\
\hline & $\geq 25 \mathrm{~K} \Gamma / \mathrm{M}^{2}$ & $181 / 45,3$ & $65 / 36,7$ & $116 / 52,0$ & 0,002 \\
\hline & $\geq 30 \mathrm{~K} \Gamma / \mathrm{M}^{2}$ & $78 / 19,5$ & $27 / 15,3$ & $51 / 22,9$ & 0,056 \\
\hline \multicolumn{2}{|c|}{ OT, cM } & $\begin{array}{c}81,71 \pm 13,45 \\
78,9(72,0 ; 89,7)\end{array}$ & $\begin{array}{c}78,86 \pm 13,23 \\
76,0(69,0 ; 86,3)\end{array}$ & $\begin{array}{c}83,97 \pm 13,22 \\
81,0(74,3 ; 92,0)\end{array}$ & $<0,001$ \\
\hline \multicolumn{2}{|c|}{ OT $\geq 80 \mathrm{~cm}, \mathrm{n} / \%$} & $189 / 47,3$ & $66 / 37,3$ & $123 / 55,2$ & $<0,001$ \\
\hline \multicolumn{2}{|c|}{ OT $\geq 88 \mathrm{~cm}, \mathrm{n} / \%$} & $110 / 27,5$ & $36 / 20,3$ & $74 / 33,2$ & 0,004 \\
\hline \multicolumn{2}{|c|}{ САД, мм рт. ст. } & $\begin{array}{c}115,96 \pm 14,68 \\
114,0(106,5 ; 122,5)\end{array}$ & $\begin{array}{c}112,60 \pm 11,00 \\
112,5(105,3 ; 120,5)\end{array}$ & $\begin{array}{c}118,61 \pm 16,59 \\
116,5(108,0 ; 126,4)\end{array}$ & $<0,001$ \\
\hline \multicolumn{2}{|c|}{ ДАД, мм рт. ст. } & $75,79 \pm 10,17$ & $\begin{array}{c}73,39 \pm 8,73 \\
73,0(66,5 ; 79,5)\end{array}$ & $\begin{array}{c}77,69 \pm 10,82 \\
76,5(70,0 ; 84,9)\end{array}$ & $<0,001$ \\
\hline \multicolumn{2}{|c|}{$\mathrm{A} \Gamma \geq 140 / 90$ мм рт.ст., n/\% } & $44 / 11,0$ & $9 / 5,1$ & $35 / 15,6$ & 0,001 \\
\hline \multicolumn{2}{|c|}{$A Г \geq 130 / 85$ мм рт.ст., n/\% } & $86 / 21,4$ & $21 / 11,9$ & $65 / 29,0$ & $<0,001$ \\
\hline \multicolumn{6}{|c|}{ Биохимические показатели, ммоль/л } \\
\hline \multicolumn{2}{|l|}{ ГПН } & $\begin{array}{c}5,61 \pm 0,64 \\
5,52(5,20 ; 5,94)\end{array}$ & $\begin{array}{c}5,48 \pm 0,60 \\
5,41(5,10 ; 5,83)\end{array}$ & $\begin{array}{c}5,71 \pm 0,66 \\
5,62(5,31 ; 6,04)\end{array}$ & $<0,001$ \\
\hline \multicolumn{2}{|l|}{ OXC } & $\begin{array}{c}4,50 \pm 0,87 \\
4,91(4,39 ; 5,50)\end{array}$ & $\begin{array}{c}4,85 \pm 0,80 \\
4,75(4,27 ; 5,27)\end{array}$ & $\begin{array}{c}5,10 \pm 0,91 \\
5,01(4,53 ; 5,65)\end{array}$ & $\begin{array}{l}0,004 \\
0,002\end{array}$ \\
\hline \multicolumn{2}{|c|}{ ХС-ЛПВП } & $\begin{array}{c}1,42 \pm 0,29 \\
1,37(1,21 ; 1,60)\end{array}$ & $\begin{array}{c}1,43 \pm 0,27 \\
1,39(1,26 ; 1,60)\end{array}$ & $\begin{array}{c}1,41 \pm 0,30 \\
1,37(1,21 ; 1,57)\end{array}$ & $\begin{array}{l}0,651 \\
0,312\end{array}$ \\
\hline \multicolumn{2}{|c|}{ ХС-лПНП } & $\begin{array}{c}3,10 \pm 0,81 \\
3,08(2,51 ; 3,59)\end{array}$ & $\begin{array}{c}2,96 \pm 0,75 \\
2,90(2,40 ; 3,47)\end{array}$ & $\begin{array}{c}3,20 \pm 0,83 \\
3,19(2,63 ; 3,65)\end{array}$ & $\begin{array}{l}0,003 \\
0,003\end{array}$ \\
\hline \multicolumn{2}{|l|}{ ТГ } & $\begin{array}{c}1,01 \pm 0,707 \\
0,85(0,63 ; 1,18)\end{array}$ & $\begin{array}{c}0,95 \pm 0,88 \\
0,76(0,60 ; 1,08)\end{array}$ & $\begin{array}{c}1,05 \pm 0,55 \\
0,93(0,69 ; 1,29)\end{array}$ & $\begin{array}{l}0,168 \\
\mathbf{0 , 0 0 1}\end{array}$ \\
\hline \multicolumn{6}{|c|}{ Биохимические показатели, n/\% } \\
\hline \multicolumn{2}{|c|}{ ГПН 6,1-6,9 ммоль/л } & $68 / 17,0$ & $22 / 12,5$ & $46 / 20,5$ & 0,032 \\
\hline \multicolumn{2}{|c|}{ ГПН 5,6-6,9 ммоль/л } & $190 / 47,5$ & $72 / 40,7$ & $118 / 52,7$ & 0,017 \\
\hline \multicolumn{2}{|c|}{ ГПН $\geq 7,0$ ммоль/л } & $6 / 1,5$ & $2 / 1,1$ & $4 / 1,8$ & 0,591 \\
\hline \multicolumn{2}{|c|}{ ОХС $\geq 5,0$ ммоль/л } & $185 / 46,3$ & $69 / 39,2$ & $116 / 51,8$ & 0,012 \\
\hline \multicolumn{2}{|c|}{ ТГ $\geq 1,7$ ммоль/л } & $39 / 10,1$ & $12 / 7,4$ & $27 / 12,1$ & 0,131 \\
\hline \multicolumn{2}{|c|}{ ХС-ЛПНП $\geq 3,0$ ммоль/л } & $207 / 53,8$ & $74 / 45,7$ & $133 / 59,6$ & 0,007 \\
\hline ХС-ЛП & ВП <1,2 ммоль/л & $90 / 23,4$ & $35 / 21,6$ & $55 / 24,7$ & 0,484 \\
\hline ХС-ЛП & ВП <1,3 ммоль/л & $139 / 36,1$ & $51 / 31,5$ & $88 / 39,5$ & 0,108 \\
\hline $\begin{array}{l}\text { Диагн } \\
\text { по крı }\end{array}$ & $\begin{array}{l}\text { остирован МС } \\
\text { териям ВНОК, } 2009\end{array}$ & $91(388) / 23,5$ & $21(166) / 12,7$ & $70(222) / 31,5$ & $<0,001$ \\
\hline $\begin{array}{l}\text { Диагн } \\
\text { по крı }\end{array}$ & $\begin{array}{l}\text { остирован MC } \\
\text { териям NCEP ATP, } 2001\end{array}$ & $64(389) / 16,5$ & $15(166) / 9,0$ & $49(223) / 22,0$ & 0,001 \\
\hline
\end{tabular}

Примечание. ИМТ - индекс массы тела; ОТ — окружность талии; САД — систолическое артериальное давление; ДАД - диастолическое артериальное давление; ГПН - глюкоза плазмы натощак; ОХС - общий холестерин; ХС-ЛПВП - холестерин липопротеинов высокой плотности; ХС-ЛПНП - холестерин липопротеинов низкой плотности; ТГ - триглицериды; МС - метаболический синдром. Значения считались статистически значимыми при $\mathrm{p}<0,05$. 
Таблица 2. Гормональные показатели и данные анкетирования женщин $25-45$ лет (M \pm SD и Me $(25 ; 75))$

\begin{tabular}{|c|c|c|c|c|}
\hline \multirow{2}{*}{ Показатели } & \multirow{2}{*}{ Вся выборка (n=401) } & \multicolumn{2}{|c|}{ Возрастная группа, лет } & \multirow{2}{*}{$\mathbf{p}$} \\
\hline & & $25-34(n=177)$ & $35-44(n=224)$ & \\
\hline ТТГ, МЕ/мл & $\begin{array}{c}1,897 \pm 2,165 \\
1,48(0,99 ; 2,19)\end{array}$ & $\begin{array}{c}1,97 \pm 2,88 \\
1,42(0,99 ; 2,05)\end{array}$ & $\begin{array}{c}1,84 \pm 1,36 \\
1,52(0,98 ; 2,28)\end{array}$ & $\begin{array}{l}0,568 \\
0,371\end{array}$ \\
\hline ПРЛ, нг/мл & $\begin{array}{c}13,62 \pm 9,60 \\
11,86(6,61 ; 17,85)\end{array}$ & $\begin{array}{c}13,14 \pm 9,39 \\
11,28(6,16 ; 17,64)\end{array}$ & $\begin{array}{c}14,00 \pm 9,76 \\
12,02(6,85 ; 17,88)\end{array}$ & $\begin{array}{l}0,373 \\
0,434\end{array}$ \\
\hline \multicolumn{5}{|c|}{$n / \%$} \\
\hline ТТГ > 4,0 МЕ/мл & $23 / 5,8$ & $11 / 6,3$ & $12 / 5,4$ & 0,712 \\
\hline ТТГ >6,1 МЕ/мл & $8 / 2,0$ & $4 / 2,3$ & $4 / 1,8$ & 0,736 \\
\hline ТТГ > 10,0 МЕ/мл & $2 / 0,5$ & $2 / 1,1$ & 0 & - \\
\hline ПРЛ $\geq 19,5$ нг/мл & $80 / 20$ & $33 / 18,6$ & $47 / 21$ & 0,561 \\
\hline Нарушение менструального цикла & $75(390) / 19,2$ & $36(173) / 20,8$ & $39(217) / 18,0$ & 0,480 \\
\hline Курит & $104 / 25,9$ & $57 / 32,2$ & $47 / 21,0$ & 0,011 \\
\hline Бросила курить & $94 / 23,4$ & $46 / 26,0$ & $48 / 21,4$ & 0,285 \\
\hline Не курит & $203 / 50,6$ & $74 / 41,8$ & $129 / 57,6$ & 0,002 \\
\hline АИТ & $25 / 6,2$ & $8 / 4,5$ & $17 / 7,6$ & 0,208 \\
\hline Зоб и/или очаговые изменения ЩЖ & $47 / 11,7$ & $21 / 11,9$ & $26 / 11,6$ & 0,937 \\
\hline ДТЗ & $5 / 1,2$ & $2 / 1,1$ & $3 / 1,3$ & 0,851 \\
\hline Операция на ЩЖ & $2 / 0,5$ & 0 & $2 / 0,9$ & - \\
\hline
\end{tabular}

Примечание. ТТГ — тиреотропный гормон; ПРЛ — пролактин; АИТ - аутоиммунный тиреоидит; ДТЗ - диффузно-токсический зоб; щЖ - щитовидная железа. Значения считались статистически значимыми при $\mathrm{p}<0,05$.

гипергликемии натощак; отмечено статистически значимое увеличение частоты МС независимо от использованного критерия.

Далее проведен анализ частоты дефиниций МС и МС В квартилях значений ПРЛ. Из анализа исключены женщины с показателями ТТГ, выходящими за границы референсных значений, т.к. ранее было показано, что проатерогенные изменения липидного спектра проявляются уже при субклиническом гипотиреозе, разрабатывается концепция о повышении уровней ТТГ как компоненте MC с ключевой ролью инсулинорезистентности $[15,16]$. Во всех квартилях ПРЛ обнаружена одинаковая частота МС. Не получено разницы в частоте АГ, гипергликемии в квартилях ПРЛ. Однако обнаружено наибольшее количество женщин с ОТ более 80 см в Q1 ПРЛ vs Q4: 55,3 vs 36,2\%, $p=0,009$ (ОШ=2,18; 95\% ДИ 1,22-3,92), как и с гиперХС-лПНП в Q1 ПРЛ vs Q4: 62,6 vs 48,9\%, p=0,063, хотя разница не достигла статистической значимости. Напротив, прирост ПРЛ ассоциируется с увеличением частоты гипоХС-ЛПВП: Q2 ПРЛ vs Q4: 16,3 vs 32,6\%, p=0,011 (ОШ=2,48; 95\% ДИ 1,23-5,02); Q2 ПРЛ vs Q4: 27,2 vs 46,7\%, $\mathrm{p}=0,007$ (ОШ=2,35; 95\% ДИ 1,27-4,35) соответственно (табл. 3).

При анализе процентного соотношения компонентов МС в квартилях ПРЛ у женщин с гиперпролактинемией (более 19,5 нг/мл) (n=74) получено, что в Q4 ПРЛ (Me=41,35 [34,78;45,88]) наибольшее количество женщин с ОТ более 80 см - 56\%, с АД $\geq 130 / 85$ мм рт.ст. - 39\%, с наличием MC - 47 и 39\% (табл. 4).

Таким образом, более неблагоприятные метаболические показатели имеют женщины с низконормальными значениями ПРЛ (Ме=4,49 [3,52; 5,41] нг/мл). МС выявлен у $28 \%$, с преимущественным увеличением частоты АО 55\%, гиперХС-лПНП - 63\%. Женщины, имеющие высокие значения ПРЛ (Ме=41,35 [34,78;45,88] нг/мл), также имеют неблагоприятный метаболический профиль: МС обнаружен у $47 \%, \mathrm{AO}-56 \%, \mathrm{AГ}-39 \%$.

\section{Дополнительные результаты исследования}

В более молодой возрастной группе больше курящих женщин, каждая третья женщина указала, что курит

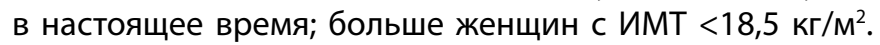
По данным опроса сопоставима частота АИТ (аутоиммунного тиреоидита), ДТЗ (диффузно-токсического зоба), структурных нарушений щитовидной железы в изучаемых возрастных группах. Каждая пятая женщина в обеих группах указала на нарушения менструального цикла.

Так как значения ПРЛ и ТТГ сопоставимы в возрастных группах 25-34 и 35-45 лет, дальнейший анализ проводился на объединенной группе женщин 25-45 лет. При проведении корреляционного анализа Спирмена получены ассоциации ПРЛ с нарушениями менструального цикла $(r=0,230, p=0,001)$; слабая положительная связь с ТТГ $(r=0,100, p=0,046)$.

Были изучены показатели ПРЛ при наличии/отсутствии МС и его компонентов у женщин 25-45 лет (табл. 5). Из анализа исключены женщины с показателями ТТГ, выходящими за границы референсных значений. Показано, что средние значения ПРЛ не различались у женщин с и без МС и при анализе отдельных компонентов МС.

Нежелательные явления

Нежелательные явления за время проведения исследования не зарегистрированы. 
Таблица 3. Процентное соотношение компонентов метаболического синдрома в квартилях пролактина у женщин в популяционной выборке 25-45 лет

\begin{tabular}{|c|c|c|c|c|c|c|c|c|}
\hline \multirow{4}{*}{ Показатели } & \multicolumn{8}{|c|}{ Квартили пролактина, Ме $(25 ; 75)$ нг/мл } \\
\hline & \multirow{2}{*}{\multicolumn{2}{|c|}{$\begin{array}{c}1 \\
(n=94) \\
35,5 \pm 5,8 \text { года } \\
4,49 \\
{[3,52 ; 5,41]}\end{array}$}} & \multirow{2}{*}{\multicolumn{2}{|c|}{$\begin{array}{c}2 \\
(n=95) \\
36,0 \pm 6,3 \text { года } \\
9,11 \\
{[7,84 ; 10,63]}\end{array}$}} & \multirow{2}{*}{\multicolumn{2}{|c|}{$\begin{array}{c}3 \\
(n=95) \\
36,4 \pm 6,5 \text { года } \\
14,36 \\
{[12,92 ; 15,79]}\end{array}$}} & \multirow{2}{*}{\multicolumn{2}{|c|}{$\begin{array}{c}\begin{array}{c}4 \\
(n=95) \\
36,8 \pm 6,4 \text { года }\end{array} \\
22,71 \\
{[19,64 ; 28,03]}\end{array}$}} \\
\hline & & & & & & & & \\
\hline & $\mathbf{n}$ & $\%$ & $\mathbf{n}$ & $\%$ & $\mathbf{n}$ & $\%$ & $\mathbf{n}$ & $\%$ \\
\hline OT $\geq 80$ cм (BHOK, 2009) & 52 & 55,3 & 44 & 46,3 & 48 & 50,5 & 34 & $36,2^{*}$ \\
\hline OT $\geq 88$ cM (NCEP ATP, 2001) & 25 & 26,6 & 30 & 31,6 & 26 & 27,4 & 20 & 21,3 \\
\hline Триглицериды $\geq 1,7$ ммоль/л & 9 & 9,9 & 11 & 12,0 & 8 & 9,0 & 8 & 8,7 \\
\hline ХС-лПВП <1,2 ммоль/л (ВНОК, 2009) & 23 & 25,3 & 15 & 16,3 & 16 & 18,0 & 30 & $32,6^{\#}$ \\
\hline ХС-ЛПВП <1,3 ммоль/л (NCEP АТP, 2001) & 36 & 39,6 & 25 & 27,2 & 26 & 29,2 & 43 & $46,7^{* *}$ \\
\hline ХС-лПНП > 3,0 ммоль/л (ВНОК, 2009) & 57 & 62,6 & 46 & 50,0 & 46 & 51,7 & 45 & $48,9 \wedge$ \\
\hline АД $\geq 130 / 85$ мм рт.ст. & 22 & 23,4 & 26 & 27,4 & 15 & 15.8 & 19 & 20,0 \\
\hline Глюкоза плазмы натощак $\geq 6,1$ ммоль/л & 15 & 16,1 & 13 & 14,1 & 17 & 17,9 & 18 & 18,9 \\
\hline Диагностирован МС по критериям ВНОК, 2009 & 26 & 28,3 & 19 & 20,7 & 20 & 22,0 & 19 & 20,9 \\
\hline Диагностирован MC по критериям NCEP ATP, 2001 & 14 & 15,2 & 16 & 17,4 & 14 & 15,4 & 16 & 17,2 \\
\hline
\end{tabular}

Примечание. ОТ - окружность талии; АД - артериальное давление; ОХС - общий холестерин; ХС-лПВП - холестерин липопротеинов высокой плотности; ХС-ЛПНП - холестерин липопротеинов низкой плотности; МС - метаболический синдром. Значения считались статистически значимыми при $\mathrm{p}<0,05$.

${ }^{*} p_{\text {отz80 см }}=0,009$, уровень статистической значимости между 1 и 4 квартилями.

" $\mathrm{p}_{\mathrm{xс-л} \text { ппвп <1,2 ммопь/ }}=0,011$, уровень статистической значимости между 2 и 4 квартилями.

${ }^{* *} p_{\text {хс-лпвп }<1,3 \text { ммоль/л }}=0,007$, уровень статистической значимости между 2 и 4 квартилями.

$\wedge \mathrm{p}_{\text {хс-лпнп >3,0 ммоль/л }}=0,063$, уровень статистической значимости между 1 и 4 квартилями.

Таблица 4. Процентное соотношение компонентов метаболического синдрома в квартилях пролактина у женщин 25-45 лет с гиперпролактинемией

\begin{tabular}{|c|c|c|c|c|c|c|c|c|c|}
\hline \multirow{3}{*}{ Показатели } & \multicolumn{9}{|c|}{ Квартили пролактина, Ме (25; 75), нг/мл } \\
\hline & \multicolumn{2}{|c|}{$\begin{array}{c}1 \\
35,6 \pm 5,3 \\
20,52 \\
{[19,81 ; 21,33]}\end{array}$} & \multicolumn{2}{|c|}{$\begin{array}{c}2 \\
38,2 \pm 6,8 \\
23,24 \\
{[22,35 ; 23,78]}\end{array}$} & \multicolumn{2}{|c|}{$\begin{array}{c}3 \\
36,3 \pm 6,7 \\
26,84 \\
{[25,45 ; 28,44]}\end{array}$} & \multicolumn{2}{|c|}{$\begin{array}{c}4 \\
38,0 \pm 6,9 \\
41,35 \\
{[34,78 ; 45,88]}\end{array}$} & \multirow[t]{2}{*}{$\mathbf{p}_{1-4}$} \\
\hline & $\mathbf{n}$ & $\%$ & $\mathbf{n}$ & $\%$ & $\mathbf{n}$ & $\%$ & $\mathbf{n}$ & $\%$ & \\
\hline OT >80 cм (BHOK, 2009) & 4 & 22,2 & 8 & 42,1 & 8 & 42,1 & 10 & $55,6^{*}$ & 0,040 \\
\hline OT >88 cM (NCEP ATP, 2001) & 2 & 11,1 & 7 & 36,8 & 3 & 15,8 & 5 & 27,8 & 0,238 \\
\hline Триглицериды $\geq 1,7$ ммоль/л & 0 & - & 0 & - & 3 & 15,8 & 5 & 29,4 & - \\
\hline $\begin{array}{l}\text { ХС-ЛПВП <1,2 ммоль/л } \\
\text { (ВНОК, 2009) }\end{array}$ & 5 & 27,8 & 7 & 41,2 & 4 & 21,1 & 6 & 35,3 & 0,586 \\
\hline $\begin{array}{l}\text { ХС-ЛПВП <1,3 ммоль/л } \\
\text { (NСЕРАТР, 2001) }\end{array}$ & 5 & 27,8 & 10 & 58,8 & 8 & 42,1 & 8 & 47,1 & 0,316 \\
\hline $\begin{array}{l}\text { ХС-ЛПНП > 3,0 ммоль/л } \\
\text { (ВНОК, 2009) }\end{array}$ & 8 & 44,4 & 12 & 70,6 & 10 & 52,6 & 7 & 41,2 & 0,312 \\
\hline АД $\geq 130 / 85$ мм рт.ст. & 1 & 5,6 & 5 & 26,3 & 5 & 26,3 & 11 & $38,9^{\#}$ & 0,016 \\
\hline $\begin{array}{l}\text { Глюкоза плазмы натощак } \geq 6,1 \\
\text { ммоль/л }\end{array}$ & 2 & 11,1 & 4 & 21,1 & 3 & 15,8 & 3 & 16,7 & 0,878 \\
\hline $\begin{array}{l}\text { Диагностирован МС } \\
\text { по критериям ВНОК, } 2009\end{array}$ & 1 & 5,6 & 5 & 29,4 & 4 & 21,1 & 8 & $47,1^{\wedge}$ & 0,005 \\
\hline $\begin{array}{l}\text { Диагностирован MC } \\
\text { по критериям NCEPATP III, } 2001\end{array}$ & 1 & 5,6 & 4 & 23,5 & 3 & 15,8 & 7 & $38,9 \#$ & 0,022 \\
\hline
\end{tabular}

Примечание. ОТ - окружность талии; АД - артериальное давление; ОХС - общий холестерин; ХС-ЛПВП — холестерин липопротеинов высокой плотности; ХС-лПНП - холестерин липопротеинов низкой плотности; МС - метаболический синдром. Значения считались статистически значимыми при $\mathrm{p}<0,05$. 
Таблица 5. Показатели пролактина при наличии/отсутствии метаболического синдрома и его компонентов у женщин 25-45 лет

\begin{tabular}{|c|c|c|}
\hline Показатели & ПРЛ (M $\pm S D)$ & $\mathbf{p}$ \\
\hline OT $\geq 80$ cм (BHOK, 2009) & $12,78 \pm 9,48$ & \multirow{2}{*}{0,154} \\
\hline OT $<80 \mathrm{~cm}$ & $14,17 \pm 9,29$ & \\
\hline OT $\geq 88$ см (NCEP ATP, 2001) & $12,87 \pm 8,89$ & \multirow{2}{*}{0,420} \\
\hline OT $<88 \mathrm{~cm}$ & $13,75 \pm 9,58$ & \\
\hline Триглицериды $\geq 1,7$ ммоль/л & $14,57 \pm 11,22$ & \multirow{2}{*}{0,473} \\
\hline Триглицериды <1,7 ммоль/л & $13,39 \pm 9.14$ & \\
\hline ХС-ЛПВП <1,2 ммоль/л (ВНОК, 2009) & $14,73 \pm 10,56$ & \multirow{2}{*}{0,172} \\
\hline ХС-ЛПВП $\geq 1,2$ ммоль/л & $13,14 \pm 8,95$ & \\
\hline ХС-ЛПВП <1,3 ммоль/л (NCEPATP, 2001) & $14,34 \pm 10,08$ & \multirow{2}{*}{0,209} \\
\hline ХС-ЛПВП $\geq 1,3$ ммоль/л & $13,05 \pm 8,92$ & \\
\hline АД $\geq 130 / 85$ мм рт.ст. & $13,77 \pm 10,71$ & \multirow{2}{*}{0,793} \\
\hline АД <130/85 мм рт.ст. & $13,46 \pm 9,01$ & \\
\hline Глюкоза плазмы натощак $\geq 6,1$ ммоль/л & $14,06 \pm 9,43$ & \multirow{2}{*}{0,663} \\
\hline Глюкоза плазмы натощак <6,1 ммоль/л & $13,49 \pm 9,43$ & \\
\hline ХС-ЛПНП \3,0 ммоль/л (ВНОК, 2009) & $13,04 \pm 9,66$ & \multirow{2}{*}{0,304} \\
\hline ХС-ЛПНП <3,0 ммоль/л & $14.05 \pm 8,99$ & \\
\hline МС по критериям ВНОК, 2009 & $13,98 \pm 11,30$ & \multirow{2}{*}{0,577} \\
\hline Лица без МС & $13,33 \pm 8,69$ & \\
\hline MC по критериям NCEP ATP, 2001 & $15,12 \pm 11,00$ & \multirow{2}{*}{0,168} \\
\hline Лица без МС & $13,28 \pm 9,12$ & \\
\hline
\end{tabular}

Примечание. ОТ - окружность талии; АД - артериальное давление; ОХС - общий холестерин; ХС-лПВП - холестерин липопротеинов высокой плотности; ХС-ЛПНП - холестерин липопротеинов низкой плотности; МС - метаболический синдром. Значения считались статистически значимыми при $\mathrm{p}<0,05$.

\section{ОБСУЖДЕНИЕ}

Данное исследование выполнено в г. Новосибирске, который является типичным для Сибири крупным индустриальным центром. Были обследованы женщины Октябрьского района - типичного для Новосибирска по производственной, социальной, популяционно-демографической, транспортной структурам и уровню миграции населения. Репрезентативная выборка женщин в возрасте 25-45 лет сформирована по таблицам случайных чисел на основе избирательных списков. Объем подвыборки определен с помощью генератора случайных чисел. Вышеизложенное позволяет экстраполировать полученные данные на целевую популяцию.

Резюме основного результата исследования

Полученные нами данные свидетельствуют, что низкие и высокие уровни ПРЛ неопухолевого генеза связаны с ухудшением метаболических показателей у женщин молодого возраста. Женщины с показателями ПРЛ на уровне нормальных, высоконормальных значений и умеренной гиперпролактинемии имеют благоприятный метаболический профиль. В настоящее время при обследовании пациенток с гиперПРЛ необходимо учитывать плейотропные эффекты ПРЛ.

Обсуждение основного результата исследования

В последние годы накапливаются сведения о том, что, помимо увеличения массы тела, патологическая гипер- пролактинемия сопровождается гиперинсулинемией, инсулинорезистентностью, АГ, дислипидемией, нарушением эндотелиальной функции, активацией медленно прогрессирующего воспаления [2-5]. Как отмечают многие авторы, метаболическим последствиям гиперпролактинемии неопухолевого генеза в течение многих лет не уделяется должного внимания.

В изучаемой выборке женщин распространенность ожирения составила 20\%, АО - 47\%, гиперХС-лПНП 54\%, гипоХС-ЛПВП- 23\%, АГ - 21\%, гипергликемии 17\%, гиперТГ - 10\%. Частота МС (ВНОК, 2009) - 23,5\%, по критериям NCEP ATP (2001) - 16,5\%. 26\% женщин курят в настоящее время. С нашими данными сопоставимы результаты, представленные ранее Рагино Ю.И. и соавт. на большей выборке, которые явились прогностически неблагоприятными в отношении кардиоваскулярной патологии среди молодых женщин г. Новосибирска [17].

По данным опроса, частота АИТ, ДТЗ, структурных нарушений щитовидной железы в изучаемых возрастных группах женщин не различалась. Каждая пятая женщина в обеих группах указала на нарушения менструального цикла. Так как средние значения и показатели медианы ПРЛ и ТТГ сопоставимы в возрастных группах 25-34 и 35-45 лет, дальнейший анализ метаболических показателей проводился на объединенной группе женщин 25-45 лет. Yazmín Macotela и соавт. (2020) предлагают для оценки метаболического состояния пациента в зависимости от уровня ПРЛ основываться 
на квартильном распределении гормона, а не на общепринятом «нормопролактинемическом диапазоне» [18]. Во всей выборке женщин наибольшая частота МC (BHOK, 2009) - $28 \%$ определена в низконормальном диапазоне

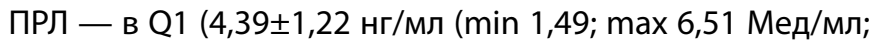
Me 4,49 (3,52; 5,41) нг/мл), с преимущественным увеличением частоты гиперХС-ЛПНП - 63\%, АО - 55\%. Ранее нами также было показано, что более неблагоприятные метаболические показатели имеют женщины с низконормальными значениями ПРЛ [19]. Наши данные сопоставимы с данными литературы, где в работах с разным дизайном исследователи показывают, что низкий уровень ПРЛ (уровни ниже 7 мкг/л) оказывает негативное влияние на обмен веществ. Две из них представляли собой большие когортные исследования у взрослых, показывающие обратную связь между уровнями ПРЛ и распространенностью СД2 и НТГ (нарушенной толерантностью к глюкозе) [20, 21]. Эти исследования были расширены и подтверждены в разных популяциях [22-25]. В третьем исследовании участвовали дети (средний возраст 10,7 года), и было обнаружено, что низкие уровни ПРЛ имели место при ожирении и были фактором риска для развития МС независимо от других факторов [26].

Концепция низкого уровня циркулирующего ПРЛ как клинического синдрома впервые появилась в 2009 г. в связи с изучением сексуальной дисфункции, при которой у пациентов мужского пола с уровнями ПРЛ в сыворотке менее 5 мкг/л был показан более высокий риск развития МС [27]. Обнаружены более низкие значения ПРЛ у пациенток с синдромом поликистозных яичников (СПКЯ), чем у пациенток без заболевания. У женщин с СПКЯ ПРЛ отрицательно ассоциируется с такими компонентами МС, как ОТ, гиперТГ и ХС-ЛПНП и с резистентностью к инсулину (HOMA-IR), обнаружена положительная связь с ХС-ЛПВП $[28,29]$. У мужчин и женщин с неалкогольной жировой болезнью печени уровни ПРЛ были ниже, чем у контрольных субъектов, и даже ниже у пациентов с тяжелым стеатозом печени по сравнению с теми, у кого болезнь была средней тяжести [30]. Кроме того, низкие уровни ПРЛ во время беременности независимо предсказывают более высокий риск развития послеродового предиабета/диабета [30].

Среди обследованных нами женщин частота МС по критериям BНОК (2009) в Q2-Q4 ПРЛ (8-28 мг/дл) составила 21-22\%, по критериям NCEP ATP (2001) 15-17\%. Как ранее нами было представлено, женщины с уровнем ПРЛ в Q2-Q4 не имеют различий в показателях глюкозы плазмы натощак, ОХС, ТГ, ХС-лПВП, ХС-ЛПНП, ИМТ, ОТ, САД, ДАД, и средние значения изучаемых показателей находятся в своих референсных диапазонах [19]. В немногочисленных исследованиях показано, что «более высокие» концентрации ПРЛ (в пределах и выше физиологического диапазона 25 мкг/л, или 525 мЕД/л), но не достигающие чрезвычайно высоких значений, способствуют благоприятному метаболическому гомеостазу [18]. Полученные в нашем исследовании данные сопоставимы с результатами исследования Framingham Heart Study, в которое для изучения ассоциации между ПРЛ в пределах нормального диапазона, изменениями в составе тела и факторами риска СС3 были отобраны участники из когорты представителей третьего поколения. После отбора с учетом критериев включения/ исключения в изучаемой подгруппе насчитывались 832 участника (335 женщин и 497 мужчин). Средний возраст женщин составил 40,2 28,7 года, исходный уровень

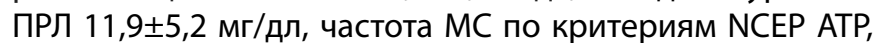
2001 - 14\%. После 2-кратного обследования с интервалом 6 лет и исключения лиц с повышенным ПРЛ (>30 мг/дл для женщин, >20 мг/дл для мужчин) исследователями показано, что уровень ПРЛ не был связан с вероятностью возникновения гиперхолестеринемии, гипертриглицеридемии или МС у женщин. В многофакторных скорректированных моделях логистической регрессии прирост базового ПРЛ на 5 мг/дл был связан с более высокими шансами низкого уровня ХС-лПВП у женщин (ОШ 1,50; 95\% ДИ 1,18-1,91; $p=0,001)$, ассоциация сохранилась после корректировки для ИМТ $(p=0,001)$. Только у женщин уровень ПРЛ был слабо положительно связан с ОХС $(r=0,05 ; p<0,05)$, однако не наблюдалось никакой связи между ПРЛ и гипертонией как одним из компонентов МС (ОШ 0,99; 95\% ДИ 0,65-1,49; $p=0,91$ ) [32].

Недавнее крупное исследование с участием более 8000 женщин (Исследование здоровья медсестер (NHS), в котором приняли участие женщины в возрасте 30-55 и 43-70 лет; в NHS II - 25-42 и 32-54 лет) показало, что более высокие, но физиологически нормальные концентрации ПРЛ были связаны с более низким риском развития сахарного диабета 2 типа в течение двух десятилетий после наблюдения и после поправки на множественные факторы риска [22]. Отличающиеся от предыдущих публикаций результаты были получены в исследовании, выполненном для оценки состояния здоровья жителей Померании (SHIP), в котором сообщается о независимой положительной связи ПРЛ, находящегося в пределах физиологической нормы, с Ме=6,4 $(4,5 ; 9,3)$ (исключили лиц с ПРЛ выше 30 мкг/л), с общей и сердечно-сосудистой смертностью. Медиана возраста женщин в этом исследовании составила 49,2 (35,9; 62,3) года и была выше, чем в нашей работе и в Framingham Heart Study. Среди женщин с уровнем ПРЛ в самом высоком тертиле (по сравнению с самым низким тертилем ПРЛ) зарегистрирован высокий риск смертности (HR 1,66; 95\% ДИ 1,08-2,56), со значительным трендом по тертилям ПРЛ (р для тренда $<0,05)$ [33]. В нашем исследовании среди женщин, имеющих высокие показатели ПРЛ, - Ме=41,35 [34,78; 45,88], но не достигающих чрезвычайно высоких значений, почти половина имеют МС - 47\%, АО - 56\%, АГ - 39\%, что свидетельствует о метаболическом нездоровье и неблагоприятном прогнозе в отношении сердечно-сосудистых заболеваний.

\section{Ограничения исследования}

Ограничением данного исследования является однократное определение уровня сывороточного ПРЛ у обследованных женщин.

Наличие пролактиномы у женщин с высокими значениями ПРЛ определялось по данным структурированного вопросника и анализа первичной медицинской документации.

\section{ЗАКЛЮЧЕНИЕ}

Значения ПРЛ от 7,8 до 28 нг/мл, т.е. условно определяемые как нормальные, высоконормальные и на уровне 
умеренной гиперпролактинемии, способствуют поддержанию благоприятного метаболического профиля. У женщин молодого возраста низкие и высокие значения ПРЛ чаще связаны с метаболическим нездоровьем. Более половины женщин с низкими и низконормальными уровнями ПРЛ имеют повышение ХС-ЛПНП (63\%) и АО (55\%). Каждая вторая женщина, имеющая высокие показатели ПРЛ неопухолевого генеза, имеет МС (47\%), AO (56\%), у 39\% наблюдается АГ. Полученные нами данные свидетельствуют о целесообразности определения ПРЛ у молодых женщин с МС, имеющих отдельные компоненты MC, такие как $A O, A Г$ и повышение уровня ХС-лПНП, с целью дальнейшего наблюдения и медикаментозной коррекции. В свою очередь, при решении вопроса о лечении женщин молодого возраста с гиперпролактинемией неопухолевой этиологии важно оценивать метаболический статус, расширяя свои представления о ПРЛ как о гормоне, связанном только с лактацией и с гипофизарно-гонадной осью.

\section{ДОПОЛНИТЕЛЬНАЯ ИНФОРМАЦИЯ}

Источник финансирования. Работа выполнена в рамках бюджетной темы по Государственному заданию No AAAA-A 17-117112850280-2 и при финансовой поддержке гранта Президента РФ для ведущих научных школ № НШ-2595.2020.7.
Конфликт интересов. Авторы декларируют отсутствие явных и потенциальных конфликтов интересов, связанных с публикацией настоящей статьи.

Участие авторов. Рымар О.Д. - разработка дизайна исследования, проверка критически важного содержания, участие в написании всех разделов и заключения, утверждение рукописи для публикации; Воевода С.М. - отбор, формирование выборки обследованных, обзор публикаций по теме статьи, анализ и интерпретация данных, написание текста рукописи; Шахтшнейдер Е.В. - участие в написании разделов материалы и методы; Стахнева Е.М. - выполнение биохимических и гормональных анализов крови, анализ данных, проверка критически важного интеллектуального содержания, участие в написании разделов материалы и методы; Мустафина С.В. - коррекция текста рукописи, проверка критически важного интеллектуального содержания; Щербакова Л.В. - формирование базы данных, статистическая обработка, анализ и интерпретация данных, участие в написании текста рукописи. Все авторы одобрили финальную версию статьи перед публикацией, выразили согласие нести ответственность за все аспекты работы, подразумевающую надлежащее изучение и решение вопросов, связанных с точностью или добросовестностью любой части работы.

Благодарности. Коллектив авторов выражает благодарность за помощь в организации проведения исследования: руководителю скрининга д.м.Н. Денисовой Д.В., руководителю НИИТПМ филиал ИЦиГ СО РАН член-корр. РАН Рагино Ю.И. и академику РАН Воеводе М.И.

\section{СПИСОК ЛИТЕРАТУРЫ | REFERENCES}

1. Soto-Pedre E, Newey PJ, Bevan JS, Greig N, Leese GP. The epidemiology of hyperprolactinaemia over 20 years in the Tayside region of Scotland: the Prolactin Epidemiology, Audit and Research Study (PROLEARS). Clin Endocrinol (Oxf). 2017;86(1):60-67. doi: https://doi.org/10.1111/cen.13156

2. Романцова Т.И. Репродукция и энергетический баланс: интегративная роль пролактина // Ожирение и метаболизм. 2014. - T. 11. - №1. - C. 5-18. [Romantsova TI. Reproduction and energy balance: the integrative role of prolactin. Obesity and metabolism. 2014;1 1(1):5-18. (In Russ.)]. doi: https://doi.org/10.14341/omet201415-18

3. Landgraf R, Landraf-Leurs MM, Weissmann A, et al. Prolactin: a diabetogenic hormone. Diabetologia. 1977;13(2):99-104. doi: https://doi.org/10.1007/BF00745135

4. Pereira-Lima JFS, Leães CGS, Neto FMF, Barbosa MV, Silva ALM, Oliveira M. Hyperprolactinemia and body weight: prevalence of obesity and overweight in patients with hyperprolactinemia. Res J Endocrinol Metab. 2013;1 (1):2. doi: https://doi.org/10.7243/2053-3640-1-2

5. Halperin Rabinovich I, Cámara Gómez R, García Mouriz M, Ollero García-Agulló D. Clinical guidelines for diagnosis and treatment of prolactinoma and hyperprolactinemia. Endocrinol y Nutr (English Ed. 2013;60(6):308-319. doi: https://doi.org/10.1016/j.endoen.2012.11.009

6. Дзеранова Л.К., Федорова Н.С., Воротникова С.Ю.,

Пигарова Е.А. Описание клинических портретов пациентов С гиперпролактинемией // Ожирение и метаболизм. - 2018. T.15. - №. 3 - C. 65-69. [Dzeranova LK, Fedorova NS, Vorotnikova SY, Pigarova EA. Description of clinical portraits of patients with hyperprolactinemia. Obesity and metabolism. 2018;15(3):65-69. (In Russ.)]. doi: 10.14341/OMET9866

7. Macotela Y, Triebel J, Clapp C. Time for a New Perspective on Prolactin in Metabolism. Trends Endocrinol Metab. 2020;31(4):276-286. doi: https://doi.org/10.1016/j.tem.2020.01.004

8. Balbach L, Wallaschofski H, Völzke H, Nauck M, Dörr M, Haring R. Serum prolactin concentrations as risk factor of metabolic syndrome or type 2 diabetes? BMC Endocr Disord. 2013;13(1):12. doi: https://doi.org/10.1186/1472-6823-13-12

9. Manshaei N, Shakibaei F, Fazilati M, et al. An investigation of the association between the level of prolactin in serum and type II diabetes. Diabetes Metab Syndr. 2019;13(5):3035-3041. doi: https://doi.org/10.1016/j.dsx.2018.07.007

10. Ponce AJ, Galván-Salas T, Lerma-Alvarado RM, et al. Low prolactin levels are associated with visceral adipocyte hypertrophy and insulin resistance in humans. Endocrine. 2020;67:331-343. doi: https://doi.org/10.1007/s12020-019-02170-x

11. Рымар О.Д., Воевода С.М., Денисова Д.В., и др. Показатели тиреотропного гормона и пролактина в популяционной выборке женщин в возрасте 25-45 лет // Доктор.Ру. - 2019. - Т. 165. №10 - C. 46-51. [Rymar OD, Voevoda SM, Denisova DV, et al. Thyroid-Stimulating Hormone and Prolactin Levels in a Populationbased Sample of Women Aged 25 to 45. DoctorRu. 2019;165(10):46-51. (In Russ.)]. doi: https://doi.org/10.31550/1727-2378-2019-165-10-46-5

12. Bland JM. An Introduction to Medical Statistics, 3rd edition. Oxford Univ Press. 2000.

13. Pasternak RC. 2001 National Cholesterol Education Program (NCEP) Guidelines on the Detection, Evaluation and Treatment of Elevated Cholesterol in Adults: Adult Treatment Panel III (ATP III). ACC Curr J Rev. 2002;1 1(4):37-45. doi: https://doi.org/10.1016/S1062-1458(02)00670-0

14. Рекомендации экспертов Всероссийского научного общества кардиологов по диагностике и лечению метаболического синдрома. Второй пересмотр // Практическая медичина. 2010. - T. 5. — №44. - C. 81-101. [Rekomendacii ekspertov Vserossijskogo nauchnogo obshchestva kardiologov po diagnostike i lecheniyu metabolicheskogo sindroma. Vtoroj peresmotr. Prakticheskaya medicina. 2010;5(44):81-101 (In Russ.)].

15. Мустафина С.В., Рымар О.Д., Симонова Г.И., и др. Функциональное состояние щитовидной железы и липидный профиль крови // Атеросклероз. - 2010. - Т. 6. — №2. - С. 15-19. [Mustafina SV, Rymar OD, Simonova Gl, et al. Functional state of thyroid gland and lipid blood profile. Ateroskleroz. 2010;6(2):15-19. (In Russ.)].

16. Руяткина Л.А., Руяткин Д.С. Субклинический гипотиреоз и метаболический синдром: основания для медикаментозного вмешательства // Ожирение и метаболизм. - 2020. T. 17 — №1. — C. 41-47. [Ruyatkina LA, Ruyatkin DS. Subclinical hypothyroidism and metabolic syndrome: reasons for drug intervention. Obesity and metabolism. 2020;17(1):41-47. (InRuss.)]. doi: https://doi.org/10.14341/omet12282 
17. Рагино Ю.И., Облаухова В.И., Денисова Д.В., и др. Абдоминальное ожирение и другие компоненты метаболического синдрома среди молодого населения г. Новосибирска // Сибирский медииинский журнал. - 2020. - T. 35. — №1. — C. 167-176. [Ragino Yul, Oblauhova VI, Denisova DV, et al. Abdominal'noe ozhireniei drugie komponenty metabolicheskogo sindroma sredi molodogo naseleniya g. Novosibirska. Sibirskij medicinskij zhurnal. 2020;35(1):167-176. (In Russ.)]. doi: https://doi.org/10.29001/2073-8552- 2020-35-1-167-176

18. Macotela Y, Triebel J, Clapp C. Time for a New Perspective on Prolactin in Metabolism. Trends EndocrinolMetab. 2020;31(4):276-286. doi: https://doi.org/10.1016/j.tem.2020.01.004

19. Воевода С.М., Щербакова Л.В., Денисова Д.В., и др. Связь атеросклероз-ассоциированных кардиоваскулярных факторов риска с разным уровнем пролактина у женщин репродуктивного возраста // Атеросклероз. - 2018. T. 14.— №4. - C. 67-72. [Voevoda SM, Shcherbakova LV, Rymar OD. Association of atherosclerosis-associated cardiovascular risk factors at different levels of prolactin in women of reproductive age. Ateroskleroz. 2018;14(4):67-72. (In Russ.)]. doi: https://doi.org/ 10.15372/ATER20180407

20. Balbach L, Wallaschofski H, Völzke H, et al. Serum prolactin concentrations as risk factor of metabolic syndrome or type 2 diabetes? BMC Endocr Disord. 2013;13(1):12. doi: https://doi.org/10.1186/1472-6823-13-12

21. Wang T, Lu J, Xu Y, et al. Circulating Prolactin Associates With Diabetes and Impaired Glucose Regulation: A population-based study. Diabetes Care. 2013;36(7):1974-1980. doi: https://doi.org/10.2337/dc12-1893

22. Li J, Rice MS, Huang T, et al. Circulating prolactin concentrations and risk of type 2 diabetes in US women. Diabetologia. 2018;61(12):2549-2560 doi: https://doi.org/10.1007/s00125-018-4733-9

23. Wang T, Xu Y, Xu M, et al. Circulating Prolactin and Risk of Type 2 Diabetes: A Prospective Study. Am J Epidemiol. 2016;184(4):295-301. doi: https://doi.org/10.1093/aje/kwv326

24. Manshaei N, Shakibaei F, Fazilati M, et al. An investigation of the association between the level of prolactin in serum and type II diabetes. Diabetes Metab Syndr. 2019:13(5):3035-3041. doi: https://doi.org/10.1016/j.dsx.2018.07.007

25. Ponce AJ, Galván-Salas T, Lerma-Alvarado RM, et al. Low prolactin levels are associated with visceral adipocyte hypertrophy and insulin resistance in humans. Endocrine. 2020;67(2):331-343. doi: https://doi.org/10.1007/s12020-019-02170-x

26. Chirico V, Cannavò S, Lacquaniti $A$, et al. Prolactin in obese children: a bridge between inflammation and metabolicendocrine dysfunction. Clin Endocrinol (Oxf). 2013;79(4):537-544. doi: https://doi.org/10.1111/cen.12183

27. Corona G, Mannucci E, Jannini EA, et al. Hypoprolactinemia: a new clinical syndrome in patients with sexual dysfunction. J Sex Med. 2009;6(5):1457-1466. doi: https://doi.org/10.1111/j.1743-6109.2008.01206.x

28. Albu A, Florea S, Fica S. Is prolactin the missing link in adipose tissue dysfunction of polycystic ovary syndrome patients? Endocrine. 2016;51(1):163-173. doi: https://doi.org/10.1007/s12020-015-0655-1

29. Glintborg D, Altinok M, Mumm H, at al. Prolactin is associated with metabolic risk and cortisol in 1007 women with polycystic ovary syndrome. Hum Reprod. 2014;29(8):1773-1779. doi: https://doi.org/10.1093/humrep/deu133

30. Zhang P, Ge Z, Wang H, et al. Prolactin improves hepatic steatosis via CD36 pathway. J Hepatol. 2018;68(6):1247-1255. doi: https://doi.org/10.1016/j.jhep.2018.01.035

31. Retnakaran R, Ye C, Kramer CK, et al. Maternal Serum Prolactin and Prediction of Postpartum $\beta$-Cell Function and Risk of Prediabetes/Diabetes. Diabetes Care. 2016;39(7):1250-1258. doi: https://doi.org/10.2337/dc16-0043

32. Therkelsen KE, Abraham TM, Pedley A, et al. Association Between Prolactin and Incidence of Cardiovascular Risk Factors in the Framingham Heart Study. J Am Heart Assoc. 2016;5(2). doi: https://doi.org/10.1161/JAHA.115.002640

33. Haring R, Friedrich $\mathrm{N}$, Völzke $\mathrm{H}$, et al. Positive association of serum prolactin concentrations with all-cause and cardiovascular mortality. Eur Heart J. 2014;35(18):1215-1221. doi: https://doi.org/10.1093/eurheartj/ehs233

\section{ИНФОРМАЦИЯ ОБ АВТОРАХ [AUTHORS INFO]:}

*Рымар Оксана Дмитриевна, д.м.н. [Oksana D. Rymar, MD, PhD]; адрес 630089, Россия, г. Новосибирск, ул. Бориса Богаткова, д. 175/1 [address: 175/1 Borisa Bogatkova street, 630089 Novosibirsk, Russia]; ORCID: https://orcid.org/0000-0003-4095-0169; eLibrary SPIN: 8345-9365; e-mail: orymar23@gmail.com

Воевода Светлана Михайловна, аспирант [Svetlana M. Voevoda, MD, postgraduate student]; ORCID: https://orcid.org/0000-0002-2908-002X; eLibrary SPIN: 4403-6722; e-mail: sm.voevoda@mail.ru

Стахнева Екатерина Михайловна, к.б.н. [Ekaterina M. Stakhneva, PhD (biology)];

ORCID: https://orcid.org/0000-0003-0484-6540; eLibrary SPIN: 7154-5094; e-mail: stahneva@yandex.ru

Шахтшнейдер Елена Владимировна, к.м.H. [Elena V. Shachtshneider, MD, PhD];

ORCID: https://orcid.org/0000-0001-6108-1025; eLibrary SPIN: 9453-9067; e-mail: 2117409@mail.ru

Мустафина Светлана Владимировна, д.м.н. [Svetlana V. Mustafina, MD, PhD]; ORCID: https://orcid.org/0000-0003-4716-876X; eLibrary SPIN: 8395-1395; e-mail: svetlana3548@gmail.ru

Щербакова Лилия Валерьевна [Lilia V. Shcherbakova]; ORCID: https://orcid.org/0000-0001-9270-9188; eLibrary SPIN: 5849-7040; e-mail: 9584792@mail.ru

*Автор, ответственный за переписку / Corresponding author.

\section{ЦИТИРОВАТЬ:}

Рымар О.Д., Воевода С.М., Шахтшнейдер Е.В., Стахнева Е.М., Мустафина С.В., Щербакова Л.В. Частота метаболического синдрома и его отдельных компонентов у женщин 25-45 лет в зависимости от уровня пролактина // Ожирение и метаболизм. - 2021. — Т. 18. — №2. — С. 180-189. doi: https://doi.org/10.14341/omet12475

\section{TO CITE THIS ARTICLE:}

Rymar OD, Voevoda SM, Shachtshneider EV, Stakhneva EM, Mustafina SV, Shcherbakova LV. The frequency of metabolic syndrome and its individual components in women aged 25-45 years, depending on the level of prolactin. Obesity and metabolism. 2021;18(2):180-189. doi: https://doi.org/10.14341/omet12475 